\title{
Redesign of a Power Slider Taking Into Account Design, Assembly and Distribution Factors
}

\author{
Jorge Rodriguez, $\mathrm{PhD} .{ }^{1}$, and Alamgir Choudhury, $\mathrm{PhD} .{ }^{1}$ \\ ${ }^{1}$ Western Michigan University, USA, jorge.rodriguez@wmich.edu
}

\begin{abstract}
Whenever an improved design is proposed or required, it is important to consider more than just the technical aspects of the new design, e.g., strength, deformation, safety, weight, cost. Nowadays it is essential to take into account several factors regarding the total production cycle of the new or improved product. Ideally every factor in the life cycle of the product should be included, but there are always limitations. In this work, in order to demonstrate the importance of other factors besides the technical one, an automotive subassembly was redesigned and evaluated. The system is a power slider assembly which is currently installed in the rear of cabin trucks and powers the rear window. The current design is bulky, expensive, and takes a long time to install. The objective was to introduce a new design for the power slider, which is more efficient in terms of operation, assembly process, and cost. A CAD model was created for the proposed design with inclusion of the new design features. Free body diagrams represented forces acting on the system, which were evaluated using finite element analysis (FEA). Based upon the results of FEA, the design will see a maximum stress of $33.9 \mathrm{MPa}$ concentrated at the lower segment of a new snap feature, which provides an acceptable safety factor. Failure mode and effect analysis (FMEA) showed potential failures and their possible causes. A prototype and cycle testing to recommended standards was performed, which provided evidence that the proposed design is ready for production. Benchmarking of proposed design versus the current design was done and significant cost savings and other benefits can be realized when all factors are taking into account. Final recommendations are presented for future evaluations. This collaboration industry-university has been a great experience and a successful one.
\end{abstract}

Keywords - Power slider, management factors, design.

Digital Object Identifier (DOI): http://dx.doi.org/10.18687/LACCEI2015.1.1.253

ISBN: 13 978-0-9822896-8-6

ISSN: $2414-6668$

$13^{\text {th }}$ LACCEI Annual International Conference: "Engineering Education Facing the Grand Challenges, What Are We Doing?" July 29-31, 2015, Santo Domingo, Dominican Republic

DOI: $\underline{\text { http://dx.doi.org/10.18687/LACCEI2015.1.1.253 }}$ 


\title{
Redesign of a Power Slider Taking Into Account Design, Assembly and Distribution Factors
}

\author{
Jorge Rodriguez, PhD, MBA, Alamgir Choudhury, $\mathrm{PhD}$ \\ Western Michigan University, Department of Engineering Design, Manufacturing, and Management Systems (EDMMS), USA \\ jorge.rodriguez@wmich.edu
}

\begin{abstract}
Whenever an improved design is proposed or required, it is important to consider more than just the technical aspects of the new design, e.g., strength, deformation, safety, weight, cost. Nowadays it is essential to take into account several factors regarding the total production cycle of the new or improved product. Ideally every factor in the life cycle of the product should be included, but there are always limitations. In this work, in order to demonstrate the importance of other factors besides the technical one, an automotive subassembly was redesigned and evaluated. The system is a power slider assembly which is currently installed in the rear of cabin trucks and powers the rear window. The current design is bulky, expensive, and takes a long time to install. The objective was to introduce a new design for the power slider, which is more efficient in terms of operation, assembly process, and cost. A CAD model was created for the proposed design with inclusion of the new design features. Free body diagrams represented forces acting on the system, which were evaluated using finite element analysis (FEA). Based upon the results of FEA, the design will see a maximum stress of 33.9 MPa concentrated at the lower segment of a new snap feature, which provides an acceptable safety factor. Failure mode and effect analysis (FMEA) showed potential failures and their possible causes. A prototype and cycle testing to recommended standards was performed, which provided evidence that the proposed design is ready for production. Benchmarking of proposed design versus the current design was done and significant cost savings and other benefits can be realized when all factors are taking into account. Final recommendations are presented for future evaluations. This collaboration industry-university has been a great experience and a successful one.
\end{abstract}

Keywords—Power slider, management factors, design.

\section{INTRODUCTION}

Current power slider assembly consists of three major components: mounting bracket housing, drum with the cable, and electric motor. This assembly is mounted on rear of current model trucks. Its function is to open and close the rear window. The current power slider has many design flaws, which can be corrected to make the design more efficient. An automotive supplier is looking for a new design that would fix the majority of the design flaws. Initial designs being proposed were rejected due to lack of analysis and testing. The proposed new design splits the mounting bracket in half, allowing the assembly to be shipped separated. Three snaps features were added to assemble the two parts.
The objective of this project was to prove that the proposed design is feasible and more effective alternative to the existing power slider assembly. In order to complete this task, background research on different aspects of the overall project was done. Different alternatives were brainstormed in order to achieve accuracy. A project plan was set up along with a fixed timeline which guided us to achieve our goal. Design validation tools were used to perform necessary tests and final results were used make further recommendations for our customer. Detail analysis on the proposed design was performed which includes free body diagrams, finite element analysis (FEA), generation of prototype parts followed by design validation cycle tests. Results obtained from the test were compared with the computer aided engineering analysis to verify the accuracy of the test. Results show the robustness of design in terms of manufacturing assembly, cycles time, maintenance cost, improved process of conveyance, and reduction of shipping cost.

\section{METHODOLOGY}

A typical design process flowchart was followed to accomplish the goal of the project. The design process is a set system in place to help engineers achieve efficient design and produce a quality product. The design process consists a chain of steps which are to identify the need, define the problem, assemble a team, apply constraints and criteria, brainstorm solutions, analyze each solution, choose the best solution, document the solution, communicate the solution, do further research, build the prototype, perform tests and finally verify and evaluate that solution. Each step is performed within budget limits.

After the redesign phase of the project, a final proposed design that is considered a solution to the current problem is provided. This new design splits the original plastic mounting bracket into two parts, thus allowing the assembly to be shipped separated, and three snaps features are added to assemble the two parts. The drum and the electric motor stay the same in the new design. Figure 1 and 2 show the existing and the proposed bracket design, respectively.

$1^{\text {th }}$ LACCEI Annual International Conference: "Engineering Education Facing the Grand Challenges, What Are We Doing?" July 29-31, 2015, Santo Domingo, Dominican Republic ISBN: 13 978-0-9822896-8-6

ISSN: $2414-6668$ 


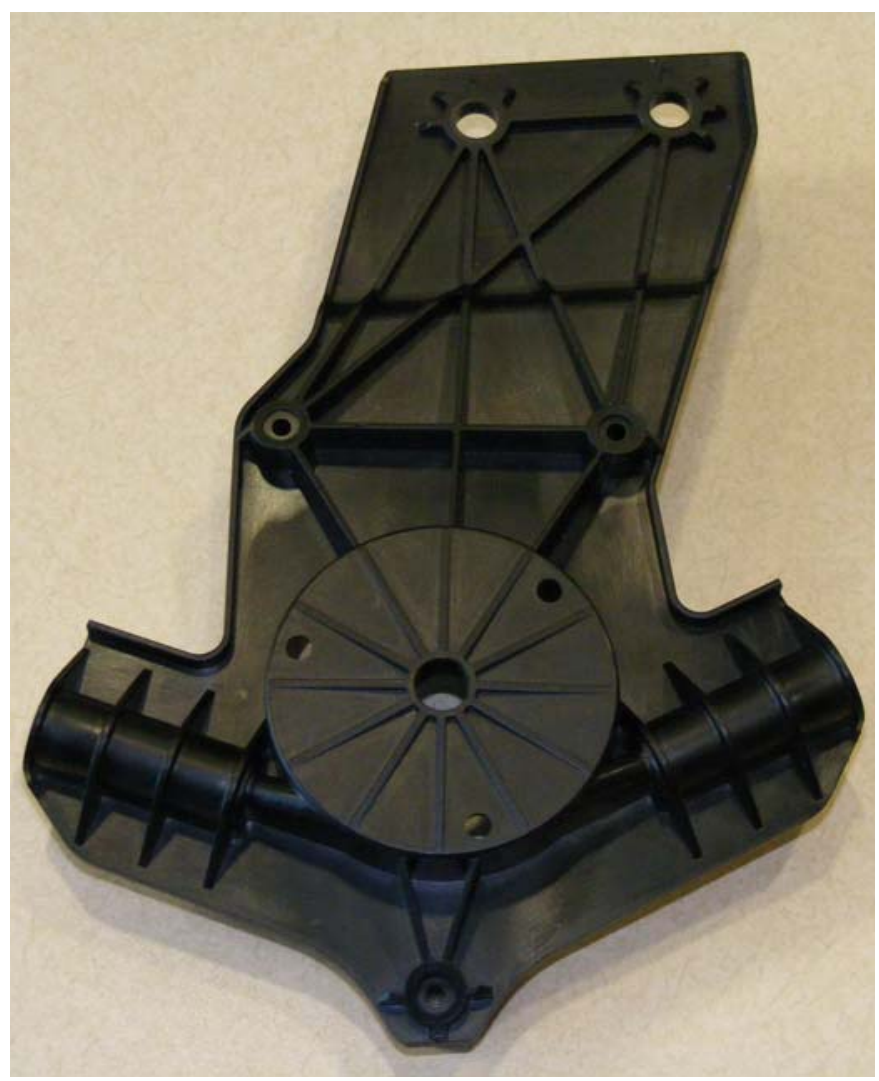

Figure 1. Current Design.

The task is now to prove that this design will work. The major steps carried out are as follows:

1. CAD modeling and Finite Element Analysis (FEA). The initial step is CAD modeling, and before FEA can begin some FBD (free body diagrams - Figure 3) were generated and evaluated in order to have proper forces specified in the FEA. The FEA was used to simulate real life operating situations on the component (Moaveni, 1999). Through this process the calculated forces were applied, we the appropriate boundary conditions.

2. Failure Mode and Effect Analysis (FMEA). FMEA is a process that is used to analyze all the different types of failure that could happen with the product (Breyfogle, 1999). This will lead to savings of time and money in the production of the component. FMEA improves the product functionality; reduces manufacturing and assembly problems, and industrial process problems. Knowing all these problems will also reduce warranty costs in the future. This is an ongoing analysis that was applied throughout the design and production process.

3. Physical prototyping. When all the initial design and analysis was completed the prototype development began (Ferreira \& Mateus, 2003). We had two possible options dealing with our prototype. One proposal was the rapid prototyping process to create our component. This is a quick process that can have a prototype ready in about a day. A negative aspect of this option is that it is not acceptable for most testing. Option number two was rapid tooling. This process uses a plastic composite to make a production die. This produces actual production parts to test and verify the design. It will also serve as a production die until metal tooling can be completed. It was critical that all design and analysis processes were carefully applied by this stage. To return to the design phase at this stage would have been detrimental to successfully completing the project. This was the most expensive phase of the project, costing several thousand dollars.

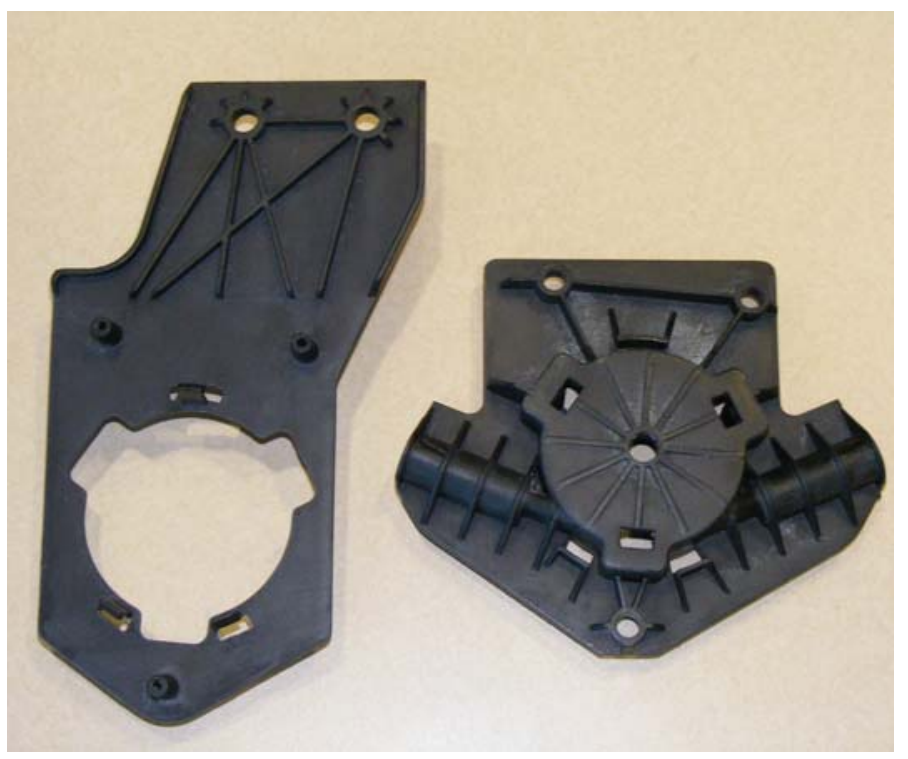

Figure 2. Proposed Design.

4. Design validation testing. The finished prototype was then subjected to design validation testing. This procedure was where the prototype was subjected to life cycle testing. We tested according to Chrysler specifications. A standard cycle uses 2 movements, open and closed. Three samples are required for testing. Each sample must go through 21,900 cycles at ambient temperature. The data collected provided us with knowledge about the safety and durability of the product. The data also served as evidence to authenticate the design.

5. Benchmarking. Benchmarking is a process that was ongoing throughout the entire project. During this phase we compared our proposed design to the existing design. This analysis included assembly time, number of parts, maintenance, and ease of assembly. The process also identifies the areas of cost such as labor, parts, and even shipping. Finalizing benchmarking marked the end of our procedure. 


\section{RESULTS}

In order to prove the effectiveness of the proposed design we worked through our established methodology. The results along with analysis and interpretation of the FEA, FMEA, rapid prototyping, design validation, and benchmarking are included in this section.

1. Modeling and Analysis. CAD modeling and finite element analysis were the first steps. FEA becomes a trial-and-error approach in order to get valid results. For both phases the software CATIA was used. For FEA, the maximum force was calculated to be 200 pounds acting through the cables. This was calculated using a GM specification of $70 \mathrm{lbs}$ stall force along with $30 \mathrm{lbs}$ of friction in the system; this was multiplied by a safety factor of 2. The maximum stress in the system is located towards the bottom of the motor mount on the inside of the snap. The magnitude of that stress is $33.9 \mathrm{MPa}$ (Figure 3 and 4). This part is made out of Polyamide 66. This material has a tensile stress at break of $210 \mathrm{MPa}$. These FEA results show that this system is operating with a static safety factor of approximately 6 . All materials specified are able to withstand a stress.

The proposed design showed no signs of failing through finite element analysis. Once that was completed the two parts were dimensioned using GD\&T. The pins and pin holes were a focused on during this process. It is important that the pins and their holes line up during assembly, if they don't the part will not fit together. A tolerance stack up was done on these two components to make sure they were dimensioned correctly. Close attention was also paid to the snap features, they must engage every time in order for the assembly to function.

2. Failure Mode and Effect Analysis. For FMEA, design and process FMEA documents (DFMEA and PFMEA) were generated. . Result of this live document is measured in terms of RPN number. RPN numbers of FMEA documents for proposed design are between 30 and 40. Industry standards indicate that RPN number less than 100 it is well within the range of safety standards. The highest RPN number was 54, and that was located in our PFMEA. That case was having a snap break when the motor bracket and drum housing are assembled. If one snap breaks the assembly is no longer a proper one. If the operator uses the pins as guides for assembly the snaps will be lined up correctly, and shouldn't break. Even though the problem is severe it has a low occurrence and is easily detectable. That is why the RPN number is below 100, and no further action in necessary. Finite element analysis and failure mode and effect analysis show that no changes are needed for the proposed design.

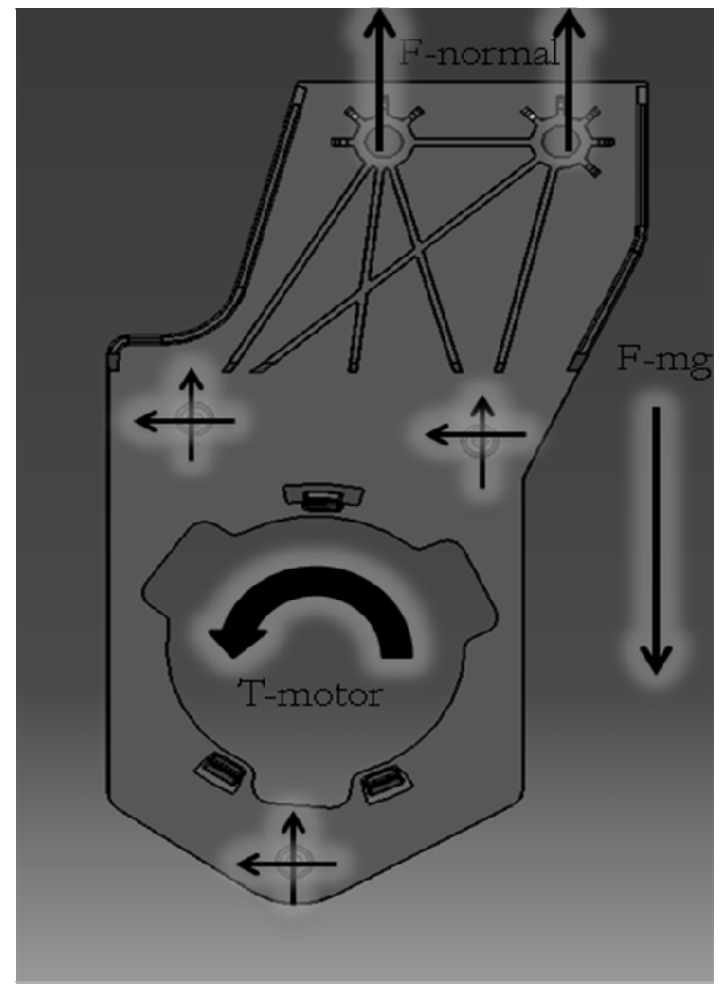

Figure 3. Free Body Diagram of Proposed Design.

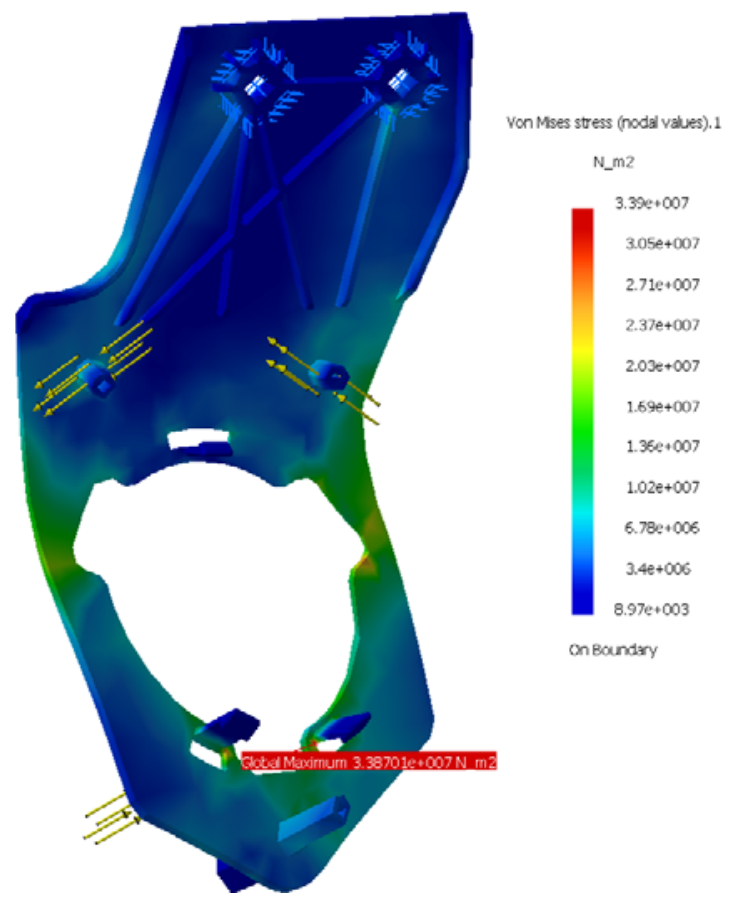

Figure 4. FEA for Proposed Design. 
3. Physical Prototyping. Production of any new design will require hundreds of thousands of dollars invested in tooling and equipment. The product needs to be able to survive durability tests for its intended use, long before tooling can be considered. To achieve this, a combination of stereolithography and rapid prototype casting has been selected to produce parts for actual testing conditions. The total discounted cost of this process was close to $\$ 3 \mathrm{k}$, which is still a small fraction of actual cost. That includes a stereolithography model of each part, two silicon molds, and ten urethane castings of each part.

4. Testing. The testing phase will further verify the results of analysis. This portion will now validate the reliability and durability of the product. This should provide the data needed to offer the product solution to major corporations for production. This testing was performed to Chrysler specifications. This phase consisted of 3 samples that were tested at ambient temperature. The samples had to survive three bogeys for the part to pass. Each bogey consisted of 7,300 cycles, which is considered one operation lifetime. Each sample will run through 21,900 cycles. Any failures to complete this process will be documented.

The first two parts passed the test, the third part only survived one operation lifetime, failing at the snap during the second set. Based on this information, an FEA sensitivity study was ran to see how the thickness of the snaps affects the stress at the snaps. These analyses showed that if the snap was increased by $1 \mathrm{~mm}$ the stress in the snap would be reduced by $32 \%$ to $23.2 \mathrm{MPa}$, and the critical point would move to the side of the part where the smallest cross section is. This change is included in the final design.

5. Benchmarking. The final step is to compare our proposed design to the design currently being used. Factors like time for assembly, number of parts, maintenance, ease of assembly, and in particular cost were considered. This step demonstrates the importance of our project and the improvements made.

The proposed design consists of one more part than the current design. This extra part comes for the fact that the motor bracket is split into two separate parts, becoming the drum housing and the motor bracket. Snaps are added to easily assemble the two parts. In this case the benefits justify the addition of one part.

When assembling the proposed design one more step is added, the drum housing and motor bracket are snapped to each other. The proposed design is just as easily assembled as the current design. Snapping the two parts together is a simple task, and is made even easier by the inclusion of three pins. The pins guide the two parts together. The proposed design does not hinder maintenance.

The real time saving comes when the shipping components are assembled. In the current design the assembly must be placed into a box. This box has to be folded and put into place by an operator. It also takes up a great deal of space in the shipping container. In our proposed design the window is shipped with the motor bracket screwed into the motor separate from the drum and drum housing snapped together. These two separate components can be taped onto the glass without being placed into a large box to secure them. The proposed design will allow for a significant cost savings for packaging and shipping. There should also be further savings when labor costs are accounted for. There will be reduction in the amount of floor space usage. Focus of this project was shipping cost and packaging savings. A cost matrix was developed to calculate the cost savings. A box is used to pack assembly of current power slider. The cost of the box is 39 cents apiece. Total cost savings due to elimination of box will be $\$ 156,000$ for 400,000 parts per year, and being as well an environmentally friendly solution. Other savings for shipping cost will be increase rack density. Currently there are 20 parts stacked in each rack which are shipped in a semi-trailer which carries 32 of those racks. A Total of 640 units are shipped in one semi-trailer, 624 semi-trailers are required to ship 400,000 units. The proposed design increases the rack density from 20 to 36 parts in each rack. This will increase the total number of assemblies in each semi-trailer from 640 to 1152 . To ship 400,000 units, 348 semi-trailers will be required. There will be $44 \%$ reduction in shipping cost which will be direct cost savings (and a benefit for the environment). Total cost of more than $\$ 600,000$ will be saved by implementation of this design (Table I). This table does not included possible savings from reduction in labor. It will cost approximately $\$ 200,000$ to for the new tooling. This cost can be recouped within the first several months of implementing the proposed design.

Table I. Total Cost Savings.

\section{Total cost savings}

\begin{tabular}{l|rr}
\hline Boxes per year & 400,000 \\
\hline Piece cost of packaging box & $\$$ & 0.39 \\
\hline Yearly savings for packaging & $\$ 156,000.00$ \\
\hline Yearly shipment savings & $\$ 445,280.00$ \\
\hline Yearly project savings & $\$ 601,280.00$ \\
\hline Retooling cost & $\$ 200,000.00$ \\
\hline
\end{tabular}




\section{CONCLUSIONS AND RECOMMENDATIONS}

This project required the effective application of all stages in the design process. The problems with the current power slider design were known, and a possible solution was given with the proposed design. That proposed design had to be validated, it had to be shown that it was an improvement over the existing design. We formulated a plan to accomplish these goals. FEA was used for the analysis of the proposed design, and FMEA to examine its functionality. In order to run real life tests our group created a prototype, and design validation testing was completed on the prototype followed by benchmarking of existing versus proposed design. The proposed design passed all of the validation tests, with the added benefit of at least $\$ 400 \mathrm{~K}$ savings a year and a green product.

The value of this project as a collaboration university-industry is that presented the opportunity to directly affect a part that may make it to market. In this project there was as well the opportunity to learn about several methodologies that are not covered in courses at school. It has been a wonderful experience for everyone involved. The results of analyses and testing show that the proposed design is a major improvement over the current design. The proposed design should be implemented as soon as possible. Further research and testing should be done to see if this design can be applied to any other window assembly, resulting is even greater benefits. Chrysler engineering is interested in this new design, and it may be used on the new models.

\section{REFERENCES}

1. Breyfogle, F. (1999). Implementing six sigma. New York, NY: John Wiley \& Sons.

2. Ferreira, J. C., \& Mateus, A. (2003). Studies of rapid soft tooling with conformal cooling channels for plastic injection molding. Journal of Materials Processing Technology, 142, 508-516. Retrieved March 13, 2008

3. How to cut time to market with FEA now (2007). 3D Vision Technologies. Retrieved March 9, 2008 from http://www.3dvision.com/FEAWhitePaper/.

4. Jantzen, H. A., \& Wiedemann, B. (1999). Strategies and applications for rapid product and process development in Daimler-Benz AG. Computers in Industry, 39, 11-25

5. Moaveni, S. (1999). Finite element analysis: Theory and applications with ANSYS. Upper Saddle River, New Jersey: Prentice Hall.

6. Process failure mode and effect analysis training (2003). NASA, Retrieved March 10, 2008, from http://process.nasa.gov/documents/pfmea.pdf

7. Tres, P. (2003). Designing plastic parts for assembly. Cincinnati, Ohio: Hanser Gardner Publications. 